\title{
Design of routing algorithm for wireless sensor networks based on clustering structure
}

\author{
Zhenghua Zhang ${ }^{\mathrm{a},{ }^{*}, \text { Zheng Gong }}{ }^{\mathrm{a}},{ }_{\text {Jie }} \mathrm{Xu}^{\mathrm{a}},{ }^{\text {Jiafeng Zhang }}{ }^{\mathrm{a}}$ \\ a Yangzhou university, 196 huayang west road,Yangzhou and 225127, China
}

*Corresponding Author: zhangzh@yzu.edu.cn

\begin{abstract}
Based on the deep research of wireless sensor network routing algorithm, proposed a MEEMLC-LEACH (More Energy-Efficient Multi-Levels Clustering LEACH) optimized routing algorithm. The main contents include: Adopting the three-level clustering method and incorporating the energy factor into the calculation formula of the electoral cluster head node, shortening the distance of data transmission between the member node and the cluster head, the cluster head node and the BS; When the initial state is completely the same, concluded the network lifetime of the MEEMLC-LEACH algorithm is about 1.4 times that of the LEACH algorithm by calculating the total energy consumption of each frame; Finally, using MATLAB to simulate the optimization algorithm by using direct routing (Direct), LEACH and MEEMLC-LEACH three routing methods, Visually shows the superiority of the optimization algorithm in balancing energy consumption and prolonging network lifetime.
\end{abstract}

Keywords: agricultural internet of things; routing algorithm; wireless sensor network

\section{Introduction}

In recent years, China's agricultural informatization process is accelerating with the introduction of concepts such as "smart agriculture", at the same time the precision agriculture characterized by automation and intelligence is an inevitable choice for current and future agricultural development. Among them, the agricultural Internet of Things based on wireless sensor networks has become a research hotspot ${ }^{(1)}$. The agricultural Internet of Things is a wireless sensor network built by a large number of wireless sensor nodes through routing algorithms. The communication data is used to transmit monitoring data to each other, and the data is collected on the detection platform. Finally, a complete agricultural information detection system was constructed.

ZigBee technology is a short-range wireless communication technology, which has been widely used in recent years because of its outstanding advantages of small size and low power consumption. In the practical application of wireless sensor network technology for agriculture, transportation, medical care, home monitoring, etc., the effective transmission of data is a core part, which is directly related to whether the entire system can be continuously monitored and operated ${ }^{(2-5)}$. Data transmission relies on efficient routing algorithms. In recent years, research on routing algorithms has never been interrupted, and LEACH algorithm (Low Energy Adaptive Clustering Hierarchy) has been widely used as a low-power adaptive algorithm ${ }^{(6-10)}$.

\section{The origin of the LEACH algorithm}

Among the clustering routing algorithms, the $\mathrm{LEACH}$ algorithm is the most representative. LEACH is essentially a complete distributed routing protocol. Therefore, it does not require global information. The algorithm gives a threshold in each cycle, and each node randomly assigns a value in the interval $[0,1]$. By comparing the value with the threshold (whether it is less than), it is determined whether the node is in the cluster head node. The successful calculation method is shown in formula (1): 


$$
T(n)=\left\{\begin{array}{cc}
\frac{p}{1-p[\operatorname{rmod}(1 / p)]} & n \in G \\
0 & \text { others }
\end{array}\right.
$$

Where $\mathrm{p}$ is the percentage of the number of cluster head nodes in the total number of nodes, which represents the possibility that any node is elected as the cluster head; $r$ is the number of rounds currently circulating ${ }^{(11-14)}$.

However, LEACH still has some shortcomings. The algorithm does not consider the residual energy of the node and the distribution and density of each node when electing the cluster head. Excessive randomness sometimes shortens the life of some nodes or selects the cluster head away from the base station, which will result in uneven energy consumption. When the number of remaining surviving nodes cannot complete the routing task, the network lifetime will also be shortened ${ }^{(15-17)}$. At the same time, LEACH can not guarantee the position and number of cluster heads per round. The formation of clusters in the basic LEACH is random, which leads to uneven distribution of clusters in the network.

\section{Proposal of MEEMLC-LEACH algorithm}

\subsection{Premise assumptions}

Since the focus of this paper is on the design of routing algorithms, in order to design the algorithm conveniently and quickly and analyze its superiority, now we make the following assumptions:(1)The energy of each node is limited.This chapter aims to design a self-organizing routing method for balanced energy and to maximize network lifetime ${ }^{(18)}$. Therefore, first assume that the initial energy of each node is a fixed value, and it will not increase during the monitoring process, so that it is convenient to observe the energy consumption of each node through simulation.(2)Each node has perceptual ability.All sensor nodes need to obtain their own location and the location of other neighboring nodes within a short distance through a corresponding mechanism (such as GPS) to calculate their own distance to the base station.(3)Each node can accurately detect its own energy level, and the power of the wireless antenna is controllable and adjustable (guarantee that it can directly communicate with the BS after adjusting to a certain power).(4)The monitoring area is an $\mathrm{N} \times \mathrm{N}$ rectangular area, and the nodes are relatively evenly distributed in the monitoring area.

\subsection{Energy consumption model}

Send the $l$ bit length data to the node with distance $d$, and the energy consumed can satisfy the formula (2):

$$
E_{T x}(l, d)=l E_{\text {elec }}+l e_{\text {amp }} d^{2}
$$

When it is necessary to send the same length of data to the same node, the energy consumed by the single-hop method is generally greater than the total energy consumed by the intermediate node ${ }^{(19)}$

When the node receives the data, the energy consumption is independent of the distance $d$, and the energy consumed by the node receiving the data from the neighboring node $l$ bit of distance $d$ satisfies the formula (3):

$$
E_{R x}(l)=l E_{\text {elec }}
$$

For each wireless node, the energy consumption of the data transceiver part accounts for a large proportion of the total energy consumption, so other parts of the energy consumption are ignored here. In this paper, only the communication energy consumption is considered in the analysis.

\section{Design of MEEMLC-LEACH algorithm}

\subsection{Clustering process of MEEMLC-LEACH algorithm}

The generation of clusters is the main process of dynamic ad hoc networks. Algorithm 1 gives the main flow of MEEMLC-LEACH algorithm. 


\begin{tabular}{|l|}
\hline Algorithm 1: MEEMLC-LEACH algorithm \\
\hline 1: Initialize each node; \\
\hline 2: Elect the cluster head node of the current layer among the \\
remaining unclustered nodes; firstly, elect the cluster head node of the \\
first layer, and the initial transmission radius of the first layer is $T R_{\text {opt }}$ \\
\hline 3: selecting member nodes of each cluster head within the \\
transmission radius of the layer; \\
\hline 4: selecting a guest node of each member node within a transmission \\
radius of the layer; \\
\hline 5: Update cluster head information; \\
\hline The main update is: layer value $n$ plus 1 , transmission radius \\
$T R$ doubled : \\
TR=TR ${ }_{\text {opt }} 2^{n}$. \\
\hline 6: Repeat steps 2-5 until the transmission radius is equal to the radius \\
of the entire monitoring network, which is cover the entire network. \\
\hline 7: Wait for the end of current round data transmission and restart the \\
next round.
\end{tabular}

Algorithm 1 MEEMLC-LEACH algorithm

\subsection{MEEMLC-LEACH algorithm data transmission process}

The data collection phase mainly includes two steps:intra-cluster transmission and data transmission between cluster heads. Algorithm 2 gives the main flow of data transmission.

\begin{tabular}{l} 
Algorithm 2: Data Transfer Process \\
1: Non-cluster head nodes within each cluster aggregate data to their \\
cluster head nodes; \\
The transmission order is: Guest node member node cluster \\
head node \\
2: Each cluster head node transmits the respective data upwards \\
according to the layer value from low to high, until it is delivered to the \\
BS. \\
\hline Finally, all monitoring data is gathered at the base station BS.
\end{tabular}

Algorithm 2 data transmission process

Step 1 mainly performs the collection and aggregation process in the cluster. Each member node collects and aggregates the data of the guest nodes in the data and the communication radius according to the predetermined time, and then sends the data to the cluster head node of the cluster $^{(20)}$.
Step 2 mainly performs data collection and aggregation between clusters. Each cluster head node collects data of the member nodes managed by itself according to a predetermined time, and aggregates the collected data and its own data to the cluster head node of the upper layer. Finally, the cluster head node collects all the data and sends it to the BS.

\subsection{MEEMLC-LEACH algorithm specific implementation method}

The routing algorithm is further described below in conjunction with the model.

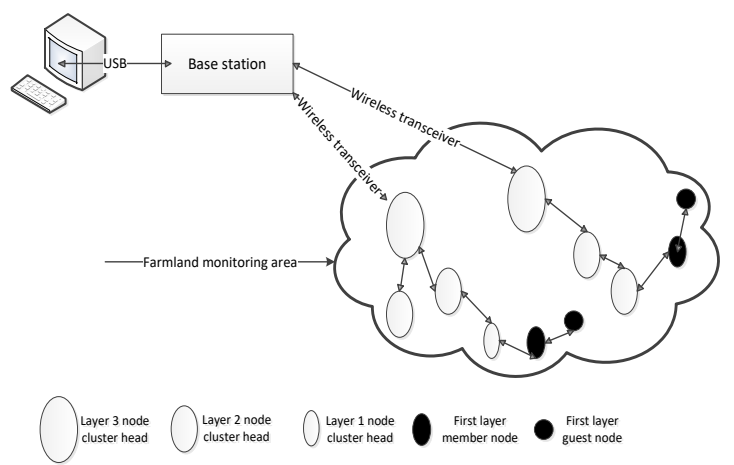

Fig.1. Schematic diagram

FIG. 1 is a schematic structural diagram of a wireless sensor network, where a wireless ad hoc network includes a plurality of aggregation nodes, a plurality of cluster head nodes, a plurality of member nodes, and a plurality of guest nodes. FIG. 1 is a schematic structural diagram of a wireless sensor network, where a wireless ad hoc network includes a plurality of aggregation nodes, a plurality of cluster head nodes, As shown in Figure 1, all nodes are layered and each layer is elected as a cluster head node. This shows that in the practical application of building a wireless network to construct an agricultural Internet of Things, it is important that the large number of nodes in the monitoring area are selforganized in a layered manner. Before the ad hoc network, all nodes need to initialize and build their own neighbor node information list. The content of the message packet broadcast by each node to the neighbor node includes: a node ID, a node residual energy RE, and a node-to-base station distance $D_{\text {toos }}$. When each node is initialized, the initial content is: the node level ClusterLevel is 0 , the cluster head node is the base station BS, the campaign cluster head result is failed, and the node transmission radius is $T R_{\text {opt }}$. 
The cluster head node of the top layer is the aggregation node. The transmission radius of the aggregation node covers the whole network. Each aggregation node uploads the merged data to the base station through wireless transmission and reception, and the base station transmits it to the upper computer for display. From the top cluster head down, each lower cluster head transfers the data of the nodes in the cluster to the cluster head in turn. This transmission mechanism can reduce the hop count during data transmission, reduce the energy consumption of the network, and shorten the transmission delay.

Each layer of nodes is divided into three levels, namely cluster head nodes, member nodes and guest nodes. Each node's message list contains its own hierarchical information. For member nodes, when they receive a cluster head generation message, first, determine whether the level in the cluster head generation message and its own level satisfies the relationship: $\mathrm{Vj}($ ClusterLevel) $+1=\mathrm{Vi}$ (ClusterLevel). If not, the member node discards directly without any response, otherwise the cluster head generation message of the cluster head node is saved in its own candidate cluster head list $\mathrm{S}_{\mathrm{CH}}$. If the member node finds that there are multiple cluster head node messages in the candidate cluster head list $\mathrm{S}_{\mathrm{CH}}$, which is there are multiple cluster heads in the neighboring node within the transmission radius then compared the ratio of the remaining energy RE of each cluster head in the candidate cluster head list $S_{C H}$ to their distance $D_{\text {toBs }}$ from the base station: $R E / D_{\text {toBs. }}$. In the end, the node with the highest ratio is successful and becomes the cluster head of the node. If there is only one cluster head node message in the candidate cluster head list $\mathrm{S}_{\mathrm{CH}}$, which is only one cluster head node in the neighbor node is within the transmission radius, the member node directly joins the cluster. For the node that has just joined and the node that is moving, if the transmission radius of the cluster head node is exceeded, these nodes are called guest nodes and indirectly join the cluster through the member nodes. after the end of the cluster head election and before the end of one round of transmission. Therefore, in addition to receiving the data of the subordinate cluster head fusion, the cluster head nodes of each layer also need to receive the data of the member nodes and the guest nodes in the cluster, after further merged and upload them to the upper cluster head

\subsection{Energy consumption analysis of MEEMLC-LEACH algorithm.}

According to the comparison of the data collection methods, both LEACH and MEEMLC-LEACH send monitoring data to the $\mathrm{BS}$ repeatedly in a frame manner at corresponding time slots. Here, we deduct and analyze the total energy consumption of the two routing methods in one frame according to the premise hypothesis and the energy consumption model. It is known that there are n nodes in the monitoring area evenly distributed in the rectangular area of $\mathrm{N} \times \mathrm{N}$, and the amount of data sent by each node takes a $l$ bit.

For LEACH, a total of $\mathrm{k}$ cluster head nodes are evenly distributed, and the probability formula of each node being elected as a cluster head node in each round is:

$$
p=\frac{k}{n}
$$

For a frame of LEACH, each cluster head node receives data sent by other member nodes in the cluster, and sends the data directly to the BS. Under the premise of uniform distribution, the number of member nodes managed by each cluster head node Satisfy formula (5):

$$
N_{n o n-C H}=\frac{n-k}{k}
$$

Therefore, the energy consumption of each cluster head node is:

$$
E_{C H}=\left(\frac{n-k}{k}\right) \cdot E_{R x}(l)+E_{T x}\left(l, d_{t o B S}\right)
$$

Substituting $\left\{\begin{array}{c}E_{R x}(l)=l E_{\text {elec }} \\ E_{T x}(l, d)=l E_{\text {elec }}+l \varepsilon_{\text {amp }} d^{2} \text { into the above }\end{array}\right.$ formula, you get:

$$
E_{C H}=l E_{\text {elec }} \frac{n}{k}+l e_{a m p} d_{t o B S}^{2}
$$

Since each node is evenly distributed in the monitoring area, the distance from the cluster head node to the base station BS is estimated as follows:

$$
E\left(d^{2}{ }_{t o B S}\right)=\int_{0}^{N} \int_{0}^{N}\left[\left(x-x_{B S}\right)^{2}+\left(y-y_{B S}\right)^{2}\right] \frac{1}{M^{2}} d x d y
$$

a frame, each non-cluster head node only needs to send data 
to the cluster head node of the cluster in which it is located, and there is no receiving task, so the energy consumption of each non-cluster head node is:

$$
E_{\text {non- } \mathrm{CH}}=E_{T x}\left(l, d_{t o C H}\right)=l E_{\text {elec }}+l e_{\text {amp }} d_{\text {toCH }}^{2}(9)
$$

The estimated distance between the non-cluster head node and the cluster head node is:

$$
E\left[d^{2}{ }_{t o C H}\right]=\frac{N^{2}}{2 p k}
$$

In summary, the energy consumption of a cluster in one frame is:

$$
E_{\text {cluster }}=E_{C H}+\left(\frac{n-k}{k}\right) \cdot E_{\text {non- } C H}
$$

Equation (12) gives the total energy consumption of the entire monitoring area in one frame:

$$
\begin{gathered}
E_{L E A C H}=k E_{\text {cluster }}(12) \\
=l\left[(2 n-k) E_{\text {elec }}+(n-k) \varepsilon_{\text {amp }} \frac{N^{2}}{2 \pi k}+k \varepsilon_{\text {amp }} E\left(d_{\text {toBS }}^{2}\right)\right]
\end{gathered}
$$

In MEEMLC-LEACH, each node shortens the distance of sending data by establishing parent-child relationship with each other. This multi-hop routing approach increases the overall energy consumption of the network in receiving data, but greatly reduces the total energy consumption in transmitting data. The energy that the entire monitoring network consumes on the received data in each frame is at most:

$$
E_{R}=(n-1) E_{R x}(l)=l(n-1) E_{\text {elec }}
$$

That is each node participates in the routing and forwarding process of data. But the energy that the non-cluster node consumes on the transmitted when sending data is:

$$
E_{T x-t o C H}=(n-k) E\left[E_{T x}\left(l, d_{t o-p a r e n t}\right)\right]
$$

Cause each sensor node is evenly distributed, the algorithm ensures that each node can find its own parent node within one hop communication radius (including the guest node looking for member nodes and member nodes looking for cluster head nodes). It is assumed here that all non-cluster nodes successfully find the parent node within the single hop range, then:

$$
p \frac{E\left(d_{t o-p a r e n t}^{2}\right)}{4} \cdot n=N^{2}
$$

which is:

$$
E\left(d_{\text {to- parent }}^{2}\right)=\frac{4 N^{2}}{p n}
$$

Therefore:

$$
E_{T x-\text { toCH }}=l(n-k)\left[E_{\text {elec }}+e_{\text {amp }} \frac{4 N^{2}}{p n}\right]
$$

Similarly, in a frame, the total energy consumed by the cluster head node in sending data to the cluster head node is:

$$
E_{T x-t o B S}=l(k-1)\left[E_{\text {elec }}+\varepsilon_{\text {amp }} \frac{4 M^{2}}{\pi k}\right]
$$

Thus, the total energy consumption consumed by the monitored area in each frame is approximately:

$$
E_{\text {MEEMLC- LEACH }}=E_{R}+E_{T x-\text { toCH }}+E_{T x-\text { toBS }}
$$

Under the premise that the initial state and the initial energy are exactly the same, when using the MEEMLC-LEACH algorithm and the LEACH algorithm, the relationship between the lifetimes of the networks is estimated as follows:

$$
\frac{T_{\text {MEEMLC. LEACH }}}{T_{\text {LEACH }}}=\frac{\frac{E}{E_{\text {MEEMLC. LEACH }}}}{\frac{E}{E_{\text {LEACH }}}}=\frac{E_{\text {LEACH }}}{E_{\text {MEEMLC. LEACH }}}
$$

\section{Simulation and Result Analysis of MEEMLC-LEACH Algorithm}

In order to evaluate the performance of MEEMLCLEACH algorithm, this paper chooses MATLAB to simulate the realization process of the designed routing algorithm. The calculation of $T(n)$ takes the formula (3), The value of the parameter $\mathrm{p}$ is $5 \%$, and the parameters used in the simulation are shown in Table 1. 
Table 1. Parameter values in the simulation

\begin{tabular}{|c|c|c|c|}
\hline parameter & value & parameter & value \\
\hline $\begin{array}{l}\text { Monitring } \\
\text { area }\end{array}$ & $\begin{array}{c}(0,0)- \\
(200,200)\end{array}$ & $\mathrm{E}_{\text {eled }} /(\mathrm{nJ} / \mathrm{bit})$ & 50 \\
\hline $\begin{array}{l}\text { Base station } \\
\text { coordinates }\end{array}$ & $(100,300)$ & $\begin{array}{c}\varepsilon_{\text {amp }} /(\mathrm{pJ} /(\text { bit.m } \\
\left.\left.{ }^{2}\right)\right)\end{array}$ & 10 \\
\hline $\begin{array}{l}\text { Total } \\
\text { number } \\
\text { ofnodes }\end{array}$ & 200 & $\begin{array}{c}\varepsilon_{\text {amp }}^{\prime} /(p J /(\text { bit.m } \\
\left.\left.{ }^{4}\right)\right)\end{array}$ & $\begin{array}{c}0.001 \\
3\end{array}$ \\
\hline $\begin{array}{l}\text { Initial } \\
\text { energy of } \\
\text { the node }\end{array}$ & 2 & $\mathrm{~d}_{0} / \mathrm{m}$ & 87 \\
\hline Packet sizel & 2000 & $\begin{array}{c}\mathrm{E}_{\mathrm{bf}} /(\mathrm{nJ} /(\text { bit.sig } \\
\text { nal)) }\end{array}$ & 5 \\
\hline
\end{tabular}

According to the value of the parameters in the table, the estimation result can be given according to the analysis result of formula (20). Compared with the traditional algorithm, the network survival time after using MEEMLC-LEACH algorithm is as shown in formula (21):

$$
\frac{T_{M E E M L C-L E A C H}}{T_{L E A C H}} \gg 1.45
$$

\section{Simulation 1: Energy-saving simulation}

Firstly, in order to test the performance of the MEEMLCLEACH algorithm in energy saving, the direct transit (Direct), LEACH and MEEMLC-LEACH routing methods are used to simulate the network lifetime. The number of nodes surviving in the three routing protocols changes as the number of frames increases (time) as shown in Figure 2.

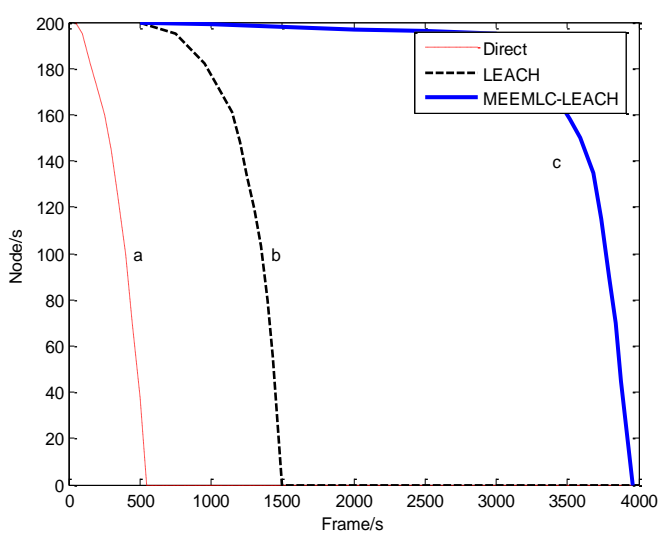

Fig.2. Comparison of the number of nodes surviving in the network

It can be seen from the simulation results of FIG. 2 that for the 200 initial nodes, when the direct transmission method is adopted, there are basically no nodes in the network after about 600 frames; When the traditional LEACH routing mode is adopted, although the nodes within 1000 frames can basically work normally, the number of nodes surviving thereafter is drastically reduced, and the network is basically paralyzed after 1500 frames.

When the MEEMLC-LEACH optimized routing algorithm designed in this paper is adopted, the entire wireless sensor network can operate normally within 3500 frames. First of all, this is because changing the longdistance single-hop route to a short-distance multi-hop route can reduce part of the energy consumption without changing the transmission distance; Secondly, the algorithm equalizes the energy consumption, so that the energy consumption speed of each node is synchronized, and some nodes are prevented from dying too quickly due to "hot spots" and the like, thereby achieving the purpose of prolonging the network life.

\section{Simulation 2: Energy Balance Performance Simulation}

In order to test the superiority of the MEEMLC-LEACH algorithm in equalizing energy consumption, the direct transmission (Direct), LEACH and MEEMLC-LEACH routing methods are used to simulate the total energy consumption of the entire network. The total network energy consumption under the three routing protocols increases as the number of frames increases (time) as shown in Figure 3.

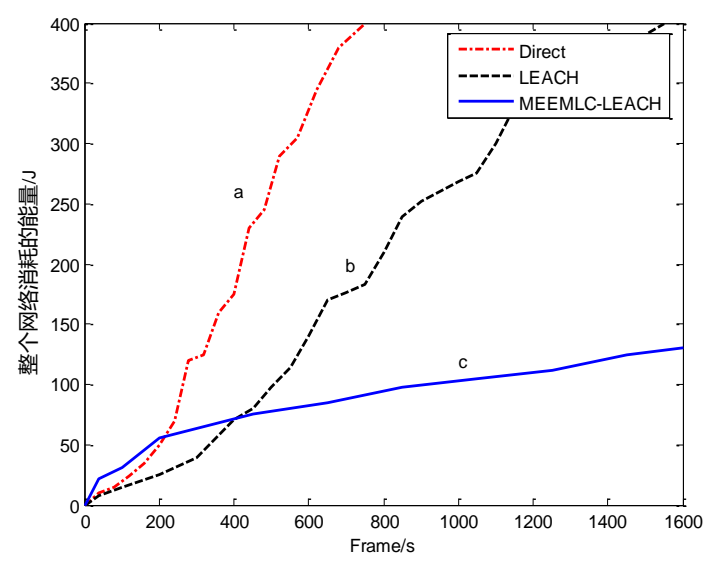

Fig.3. Comparison of energy consumption of the network

It can be seen from the simulation results in Fig. 3 that in the initial stage, the MEEMLC-LEACH algorithm consumes 
more energy than the traditional algorithm, because the algorithm needs to consume a certain amount of energy in sensing and acquiring its own position and neighbor node location and region division. However, after 200s, the improved algorithm has some improvement compared with the other two methods. This is because the optimization algorithm adopts the three-level clustering and multi-layer transmission method, which greatly shortens the communication distance of each node to transmit data.

It can be seen from the simulation results that the MEEMLC-LEACH algorithm has certain optimization and improvement in reducing energy consumption and balancing energy consumption, effectively extending the life of wireless sensor networks.

\section{Conclusion}

This paper proposes MEEMLC-LEACH (More EnergyEfficient Multi-Levels Clustering LEACH) optimized routing algorithm. The multi-layer clustering routing mechanism makes the replacement of the cluster head nodes more reasonable, and the energy consumption is maximized by minimizing the clusters. When campaigning for the cluster head node, the MEEMLC-LEACH algorithm improves the threshold calculation by taking the energy factor into consideration, which makes the election of the cluster head node more reasonable and further prolongs the survival time of the whole network.

\section{References}

(1) L.CUI,H.L.HAN, et al. Research progress in wireless sensor networks [J]. Computer Research and Development, 2005,42(1): 163-174.

(2) Heinzelman W. Application Specific Protocol Architectures for Wireless Net-works[D]. MIT.2000.

(3)Antonis Tzounis,Nikolaos Katsoulas, Thomas Bartzanas,Constantinos Kittas. Internet of Things in agriculture, recent advances and future challenges[J]. Biosystems Engineering, 2017,164.

(4) Rakesh Ranjan Swain,Pabitra Mohan Khilar,Sourav
Kumar Bhoi. Heterogeneous fault diagnosis for wireless sensor networks[J]. Ad Hoc Networks,2018,69.

(5) Ahmed Al-Baz,Ayman El-Sayed. A new algorithm for cluster head selection in LEACH protocol for wireless sensor networks $[\mathrm{J}]$. International Journal of Communication Systems, 2018,31(1).

(6)Alexander Cherepanov,Igor Tyshchenko,Mariia Popova,Dmitriy Vakhnin. Building Energy Efficient Wireless Sensor Networks[J]. International Journal of Electronics and Telecommunications,2017,63(1).

(7)Carlos Abreu,Francisco Miranda,P.M. Mendes. Smart context-aware QoS-based admission control for biomedical wireless sensor networks[J]. Journal of Network and Computer Applications,2017,88.

(8)H.LUO,Y.,.ZOU,J.X.LU,Z.H.YU,et.al. Research on Multi-parameter Routing Equilibrium Mechanism in Wireless Sensor Networks[J].Computer Science and Applications,2017,07(08).

(9) Rocío Arroyo-Valles,Andrea Simonetto,Geert Leus. Consistent sensor, relay, and link selection in wireless sensor networks[J]. Signal Processing,2017,140.

(10) Osama Moh'd Alia. Dynamic relocation of mobile base station in wireless sensor networks using a cluster-based harmony search algorithm[J]. Information Sciences,2017,385-386.

(11) Wen Zhi Zhu,Feng Xu. Minimizing Energy Consumption in Wireless Sensor Networks by Partition[J]. Applied Mechanics and Materials,2016,4450(850).

(12) Manel Souissi,Aref Meddeb. Optimal load balanced clustering in homogeneous wireless sensor networks[J]. International Journal of Communication Systems,2017,30(10).

(13) Y.SUN,H.D.MA,L.LIU, A Service Sensor Perceptual Routing Algorithm Based on Ant Colony Optimization for Multimedia Sensor Networks[J]. 2007,35(4):705-711.

(14) Huseyin Ugur Yildiz,Kemal Bicakci,Bulent Tavli,Hakan Gultekin,Davut Incebacak. Maximizing Wireless Sensor Network lifetime by communication/computation energy optimization of nonrepudiation security service: Node level versus network level strategies[J]. Ad Hoc Networks,2016,37.

(15)Hela Maddar,Wafa Kammoun,Habib Youssef. Cloudlets Architecture for Wireless Sensor Network[M].Springer International Publishing:2017-06-15.

(16)W.S.LUO,Y.P.QU,Q.LU, Research on Wireless 
Multimedia Sensor Networks[J].Journal of Electronics \& Information Technology2008,30(6): 1511-1516. (17)Chirihane Gherbi,Zibouda Aliouat,Mohamed Benmohammed. An adaptive clustering approach to dynamic load balancing and energy efficiency in wireless sensor networks[J]. Energy,2016,114.

(18)Mahdi Arghavani, Mohammad Esmaeili, Maryam Esmaeili, Farzad Mohseni, Abbas Arghavani. Optimal energy aware clustering in circular wireless sensor networks[J]. Ad Hoc Networks,2017,65.

(19)Deyu Zhang,Zhigang Chen,Haibo Zhou,Long Chen,Xuemin (Sherman) Shen. Energy -balanced cooperative transmission based on relay selection and power control in energy harvesting wireless sensor network[J]. Computer Networks,2016, 104.

(20)Y.SUN,H.D.MA, QoS guarantee problem of wireless multimedia sensor network[J].Journal of Electric Society.2008,36(7):1412 -1420. 